\title{
An Algorithm of 0-1 Knapsack Problem Based on Economic Model
}

\author{
Yingying Tian ${ }^{1}$, Jianhui Lv$^{2}$, Liang Zheng ${ }^{3}$ \\ ${ }^{1}$ School of Information Science and Technology, Sun Yat-sen University, Guangzhou 510006, China \\ ${ }^{2}$ College of Information Science and Engineering, Northeastern University, Shenyang 110819, China \\ ${ }^{3}$ School of Information Science and Technology, Sun Yat-sen University, Guangzhou 510006, China \\ Email: zlyang03@126.com, lvjianhui2012@163.com, aqlongying@163.com
}

Received August 2013

\begin{abstract}
In order to optimize the knapsack problem further, this paper proposes an innovative model based on dynamic expectation efficiency, and establishes a new optimization algorithm of 0-1 knapsack problem after analysis and research. Through analyzing the study of 30 groups of 0-1 knapsack problem from discrete coefficient of the data, we can find that dynamic expectation model can solve the following two types of knapsack problem. Compared to artificial glowworm swam algorithm, the convergence speed of this algorithm is ten times as fast as that of artificial glowworm swam algorithm, and the storage space of this algorithm is one quarter that of artificial glowworm swam algorithm. To sum up, it can be widely used in practical problems.
\end{abstract}

Keywords: 0-1 Knapsack; Economic Model; Optimization Algorithm; Storage Space

\section{Introduction}

In recent years, many experts and scholars have carried on the thorough research. These existing methods include: greedy algorithm, bound algorithm, backtracking algorithm, dynamic programming algorithm, e-approximation algorithm [1-4] and improved swarm algorithm, ant colony algorithm, fireflies swarm algorithm [5-7]. These algorithms are based on mathematical principles, since different algorithm uses different mathematical model, the complexity and the convergence speed of these algorithms are different. Thus, it has great significance to improve the convergence speed. Now 0-1 knapsack problem can be described with more details as follows:

For 0-1 knapsack problem, the object is loaded into backpack or not. The value determines whether the object $i$ is in the backpack or not. If $x_{i}=1$, the object is in the backpack, otherwise, it is not. There are the following constraint equation and objective function through analysis of 0-1 knapsack problem.

$$
\begin{gathered}
\sum_{i=1}^{n} w_{i} x_{i} \leq M \\
\text { optp }=\max \sum_{i=1}^{n} c_{i} x_{i}
\end{gathered}
$$

Among them, $M$ is deadweight of backpack, $w_{i}$ is weight of object, and $c_{i}$ is price of object. The ultimate goal is to look for the solution vector $X=\left(x_{1}, x_{2}, \ldots, x_{n}\right)$ which meets constraint Equation (1) and makes the ob- jective function value reach its maximum.

\section{Decision Algorithm}

\subsection{Ideas Origin of Algorithm}

In the general algorithm for solving the knapsack problem, these objects are arranged by their price-performance ratio in descending order. If these objects of higher price-performance ratio are loaded into the backpack in advance, the residual deadweight of backpack can converge to 0 quickly and the value of objects loaded into backpack can achieve maximum as far as possible, which is the basic idea of greedy algorithm. Solution achieved by greedy algorithm is optimal in local situation, but not optimal in global situation. It suggests that when the number of objects loaded into backpack in a certain range - before the residual deadweight of backpack is 0 - the solution is optimal in local backpack situation. This paper continues using greedy strategy.

Expectation efficiency [8] has a wide range of applications in commodity economy and stock exchange, it means that when the event occurs normally, we can expect to get revenue efficiencies. In the process of expectation, deadweight of backpack, the number of objects, weight of individual object, price of individual object and price-performance ratio of individual object all involve in arithmetic. For the same 0-1 knapsack problem, if any of four factors remain unchanged and the other factor changes, all these objects that will be loaded backpack 
and the optimal solution basically change. An abstract expectation model which is closely related into 5 factors is given through the above analysis, as follows:

$$
f(M, n, w, c, r) \infty M, n, w, c, r
$$

Those objects of higher price-performance ratio are loaded into backpack in advance, the others do expectation efficiency operation according to the model (3). These objects that have higher expectation efficiency value are loaded backpack in advance, and the lower is abandoned or delayed. The embodiment of model (3) is the key link, it decides directly the quality of algorithm.

Algorithm ideas as follows:

(a) Greedy algorithm is very good and can attain the optimal solution in solving the local problem. These first $n / 2$ objects are loaded into backpack in advance, if the total weight of $n / 2$ objects is beyond $M$, first $n / 4$ objects are selected to load into backpack in advance, according to dichotomy like this way until the total weight of $n / 2^{i}$ (i is the frequency of dichotomy) objects is no more than $\mathrm{M}$. And then, for these remaining objects, calculate the expectation efficiency.

(b) After using greedy strategy, the residual deadweight of backpack and the total value of objects loaded into backpack need to focus on. The purpose of expectation is to ensure the residual deadweight of backpack can converge to 0 quickly and the value of objects loaded backpack reach the bigger as much as possible.

\subsection{Establishment of Mathematical Model}

It is necessary to balance the five key factors when modifying this model. Referred to the theory of median and average, we design a mathematical model and show in Formula (4):

$$
\begin{aligned}
& f_{i}(M, n, w, c, r)= \\
& \frac{r_{i}}{r_{i-1}}\left|\frac{\left(\operatorname{optp}(i)-\sum_{j=1}^{\lfloor n / 2\rfloor} c_{j}-\sum_{j=\lfloor n / 2\rfloor+1}^{i} c_{j-1} x_{j-1}\right)-(n-i+1) c_{i} \mid}{\left(M-\sum_{j=1}^{\lfloor n / 2\rfloor} w_{j}-\sum_{j=\lfloor n / 2\rfloor+1}^{i} w_{j-1} x_{j-1}\right)-(n-i+1) w_{i}}\right|
\end{aligned}
$$

Among them, optp(i) is equal everywhere, so model (4) is a static model. If model (4) is taken as a dynamic expectation efficiency model, optp(i) can't be set equal everywhere. After modifying model (4), a dynamic model is given in Formula (5):

$$
\begin{aligned}
& f_{i}(M, n, w, c, r)= \\
& \frac{r_{i}}{r_{i-1}}\left|\frac{r_{i-1}\left(M-\sum_{j=1}^{\lfloor n / 2\rfloor} w_{j}-\sum_{j=\lfloor n / 2\rfloor+1}^{i} w_{j-1} x_{j-1}\right)-(n-i+1) c_{i} \mid}{\left(M-\sum_{j=1}^{\lfloor n / 2\rfloor} w_{j}-\sum_{j=\lfloor n / 2\rfloor+1}^{i} w_{j-1} x_{j-1}\right)-(n-i+1) w_{i}}\right|
\end{aligned}
$$

Some analyses are provided to illustrate model (5) in next three respects.

(a) Ending condition of dynamic model. Generally, when

$$
M-\sum_{j=1}^{\lfloor n / 2\rfloor} w_{j}-\sum_{j=\lfloor n / 2\rfloor+1}^{i} w_{j-1} x_{j-1}=0
$$

the residual deadweight of backpack is 0 , namely, it is ending condition of dynamic.

(b) Ending condition of algorithm. For these existing $f_{i}(M, n, w, c, r)$, arrange them by their values in descending order, and then to accumulate the price of the corresponding object one by one, until the backpack fails to hold on one more object.

(c) Special rule of algorithm. The average of value according to greedy expectation is always greater than the price of current object, in order to avoid the phenomenon, the algorithm has a rule: the symbol of absolute value should be added to model (5) in case the expectation efficiency values of some objects are minus. These objects, whose expectation efficiency values are minus should be removed in advance. Namely, their corresponding prices don't accumulate any more.

\subsection{Dispersion Coefficient}

Given these individual data, they are $y_{1}, y_{2}, \ldots y_{n}$, their dispersion coefficient is shown in Formula (6).

$$
v_{\sigma}=\frac{\sqrt{\sum_{i=1}^{n} \frac{\left(y_{i}-\bar{y}\right)^{2}}{n}}}{\bar{y}}
$$

In this paper, we compute three kinds of dispersion coefficient by Formula (6). Among them, $v_{\sigma}$ is dispersion coefficient, $n$ is the number of objects.

\section{Description and Analysis of Algorithm}

An optimization algorithm of 0-1 knapsack based on dynamic expectation efficiency can be descripted as follows:

Step 1: Initially, the residual deadweight of backpack is $\mathrm{M}$ and is 0 .

Step 2: Arrange these objects by their price-performance ratio in descending order.

Step 3: Modify the residual deadweight of backpack and optp according to algorithm idea (a).

Step 4: Calculate $f_{i}(M, n, w, c, r)$ of the corresponding object, if the condition of model 5 (c) is satisfied, $x_{i}=0$; otherwise, to step 5 .

Step 5: If the condition of model 5 (a) is satisfied, to step 6; otherwise, to step 4.

Step 6: If the condition of model 5 (b) is satisfied, to step 7; otherwise, to continue loading the object which has the higher $f_{i}(M, n, w, c, r)$. 
Step 7: Accumulate the price of the corresponding object, and then modify the residual deadweight of backpack and optp.

Step 8: End the algorithm.

The flowchart of DEE algorithm is shown in Figure 1.

\section{Simulation Experiment}

We present simulation experiments by writing $\mathrm{C}++$ programs with VC6.0, and the experimental environment is Windows7 of Intel Core2 Q8400 2.66GHZ CPU, 4.00GB Memory. Two simulation experiments are given, one is individual experiment of algorithm and analyzes optimal solution by three kinds of dispersion coefficient, and the other is comparison experiment with the artificial glowworm swarm optimization (GSO).

\subsection{Individual Experiment}

Given examples A and B, two kinds of dispersion coefficient are in the same level with example $\mathrm{A}$, and any kind of dispersion coefficient is not in the same level with example B. Among them, three kinds of dispersion coefficient are the weight dispersion coefficient (WDC), the price dispersion coefficient (PDC) and the price-performance ratio dispersion coefficient (RDC).

A. $n=20, M=550, w=\{45,27,58,25,33,42,55,62$, $19,72,80,24,59,35,14,66,45,83,71,40\}, \mathrm{v}=\{93,38$, $90,30,56,43,49,77,36,99,120,70,48,70,38,89,29$, $94,62,80\}$. The values of three kinds of dispersion coefficient are $0.4239,0.3943$ and 0.3955 , it' obvious that

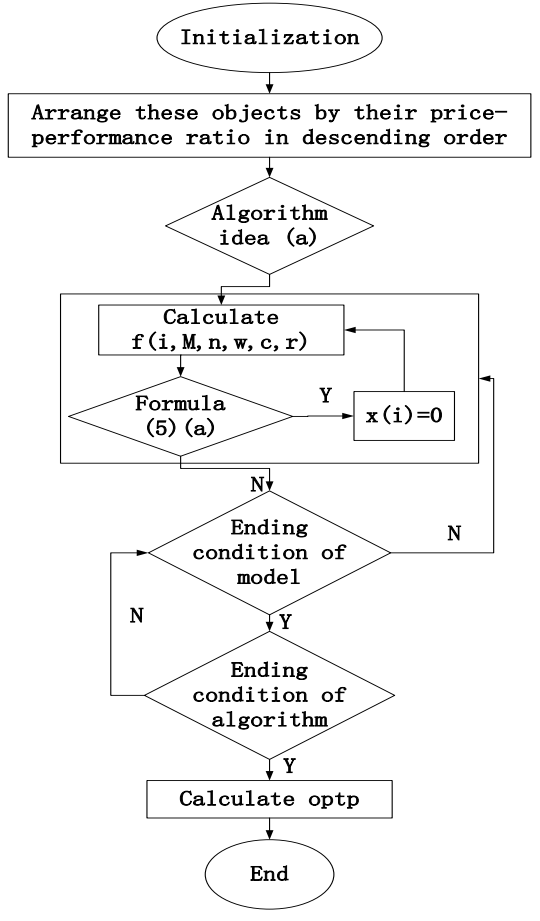

Figure 1. Process flow diagram based on DEE.
PDC and RDC are in the same level. The objective function values are equal before the 15th iteration in comparison with dynamic expectation model and static expectation model. The objective function value remains unchanged from the 15th iteration to the 20th iteration by dynamic expectation model.

B. $n=50, M=1500, w=\{10,25,30,68,74,55,36$, $120,80,95,35,45,70,85,105,48,24,15,40,50,66,58$, $28,11,20,18,38,42,28,22,32,49,60,8,78,44,52,23$, $39,19,68,99,92,77,110,69,59,43,135,136\}, v=\{20$, $48,54,118,125,92,69,185,120,140,50,60,88,105$, $128,58,29,18,46,57,74,65,31,12,21,18,36,39,26$, $20,29,43,51,6,59,32,34,22,10,34,40,28,18,16,10$, $7,5,13,12\}$. The objective function values are equal before the 49th iteration in comparison with dynamic expectation model and static expectation model.

Two experiment results show in Figure 2.

\subsection{Algorithm Proving}

In order to do further research on the adaptive scope of DEE algorithm, this paper gives 30 0-1 knapsack problems [9]. Set the number of objects is 20 , and the deadweight of backpack is in $[500,950]$. After executing DEE algorithm, the experiment results show in Table 1 in the latter.

As can be seen from Table 1, the adaptive scope of DEE algorithm is as follows: all the condition that any two kinds of dispersion coefficient are in the same level; the partial condition that all the three kinds of dispersion coefficient are less than 1 and any two kinds of dispersion coefficient are not in the same level. However, the condition that one kind of dispersion coefficient is greater than 1 and the other two kinds of dispersion coefficient are less than 1 and not in the same level is dead zone of DEE algorithm.

\subsection{Comparison Experiment with GSO Algorithm}

This part of experimental data is got by example 1 and 3 of reference [9]. The experiment results of comparison on DEE algorithm and GSO algorithm at iteration number, the optimal solution and the spaces/the number of fireflies are shown in Table 2.

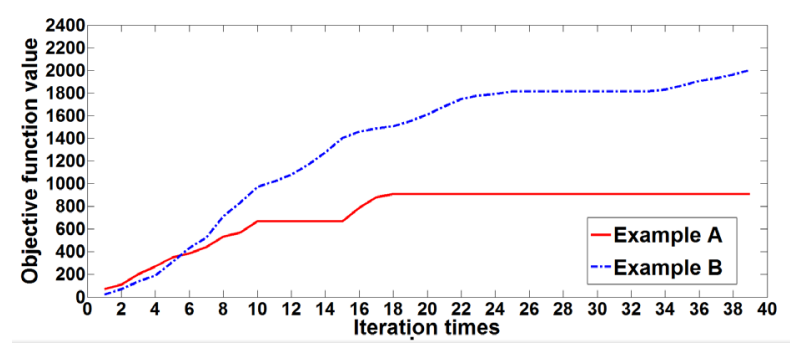

Figure 2. Experiment diagram on example $\mathrm{A}$ and $\mathrm{B}$. 
Table 1. Effect on the optimal solution by WDC, PDC, and RDC.

\begin{tabular}{|c|c|c|c|c|c|c|c|c|c|c|c|}
\hline NO. & & 1 & 2 & 3 & 4 & 5 & 6 & 7 & 8 & 9 & 10 \\
\hline & W & 0.6238 & 0.3362 & 0.7938 & 0.4437 & 0.7253 & 0.7264 & 0.5436 & 0.6215 & 0.3544 & 0.4293 \\
\hline \multirow[t]{2}{*}{$\begin{array}{l}\text { Dispersion } \\
\text { coefficient }\end{array}$} & C & 0.6254 & 0.3526 & 0.7847 & 0.3675 & 0.6381 & 0.5298 & 0.4849 & 0.6358 & 0.3570 & 0.4586 \\
\hline & $\mathrm{r}$ & 0.5360 & 0.4759 & 0.6264 & 0.3528 & 0.5124 & 1.0017 & 0.4633 & 0.7241 & 1.0211 & 0.8315 \\
\hline \multicolumn{2}{|l|}{ Objective solution } & 1205 & 869 & 1082 & 1134 & 788 & 1035 & 824 & 998 & 1336 & 1154 \\
\hline \multicolumn{2}{|l|}{ Optimal solution } & 1205 & 869 & 1082 & 1134 & 788 & 1047 & 824 & 998 & 1336 & 1154 \\
\hline \multicolumn{2}{|l|}{ Difference } & 0 & 0 & 0 & 0 & 0 & 12 & 0 & 0 & 0 & 0 \\
\hline \multicolumn{2}{|l|}{ NO. } & 11 & 12 & 13 & 14 & 15 & 16 & 17 & 18 & 19 & 20 \\
\hline & $\mathrm{w}$ & 0.7325 & 0.8576 & 0.7765 & 0.4901 & 0.7379 & 0.5348 & 0.5391 & 0.5149 & 0.3426 & 0.4993 \\
\hline \multirow[t]{2}{*}{$\begin{array}{l}\text { Dispersion } \\
\text { coefficient }\end{array}$} & C & 0.6552 & 0.6371 & 0.6082 & 0.4391 & 0.4683 & 0.4655 & 0.5296 & 0.5239 & 0.3245 & 0.4779 \\
\hline & $\mathrm{r}$ & 0.7925 & 0.7096 & 0.4367 & 0.3562 & 0.3328 & 0.5219 & 0.5417 & 0.3373 & 0.3313 & 0.0557 \\
\hline \multicolumn{2}{|l|}{ Objective solution } & 1045 & 1282 & 1420 & 1059 & 1395 & 1198 & 790 & 613 & 848 & 632 \\
\hline \multicolumn{2}{|l|}{ Optimal solution } & 1045 & 1284 & 1420 & 1059 & 1407 & 1198 & 790 & 613 & 848 & 632 \\
\hline \multicolumn{2}{|l|}{ Difference } & 0 & 2 & 0 & 0 & 12 & 0 & 0 & 0 & 0 & 0 \\
\hline \multicolumn{2}{|l|}{ NO. } & 21 & 22 & 23 & 24 & 25 & 26 & 27 & 28 & 29 & 30 \\
\hline \multirow{3}{*}{$\begin{array}{l}\text { Dispersion } \\
\text { coefficient }\end{array}$} & $\mathrm{w}$ & 0.5148 & 0.5424 & 0.6374 & 0.5403 & 0.6232 & 0.4842 & 0.6068 & 0.6315 & 0.4239 & 0.5427 \\
\hline & C & 0.4672 & 0.4952 & 0.5319 & 0.4453 & 0.5259 & 0.4552 & 0.4618 & 0.6065 & 0.3943 & 0.4810 \\
\hline & $\mathrm{r}$ & 0.4254 & 0.3523 & 1.4796 & 0.4697 & 0.7305 & 0.5169 & 0.4744 & 1.0589 & 0.3955 & 1.0118 \\
\hline \multicolumn{2}{|l|}{ Objective solution } & 973 & 843 & 783 & 1062 & 709 & 833 & 969 & 848 & 909 & 1009 \\
\hline
\end{tabular}

Table 2. The comparison results of GSO algorithm and DEE algorithm.

\begin{tabular}{|c|c|c|c|c|c|c|}
\hline \multirow{2}{*}{$\begin{array}{l}\text { Algorithm/ } \\
\text { examples }\end{array}$} & \multicolumn{3}{|c|}{ Example 1} & \multicolumn{3}{|c|}{ Example 3} \\
\hline & $\begin{array}{c}\text { The max iteration } \\
\text { number }\end{array}$ & Optimal solution & Number of fireflies/Spaces & $\begin{array}{c}\text { The max iteration } \\
\text { number }\end{array}$ & Optimal solution & $\begin{array}{c}\text { Number of } \\
\text { fireflies/Spaces }\end{array}$ \\
\hline GSO & 100 & 295 & 30 & 400 & 3103 & 100 \\
\hline DEE & 11 & 295 & 7 & 42 & 3103 & 27 \\
\hline
\end{tabular}

After the comparison of GSO algorithm and DEE algorithm, the relation of objective function value and iteration number can be described as Figures 3 and 4.

For DEE algorithm in Figures 3 and 4, the part that red line rises perpendicularly is greedy strategy and the other part that red line grows steady is dynamic expectation efficiency. The objective function value by GSO algorithm changes steady and slowly. To sum up, the average convergence speed of DEE algorithm is as 10 times fast as GSO algorithm's; the number of storage space of DEE algorithm is a quarter of GSO algorithm's.

\section{Conclusion}

Two groups of experiments show that the proposed algo-

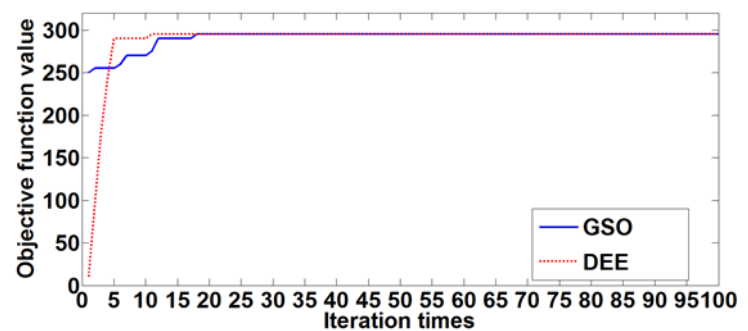

Figure 3. The convergence of GSO and DEE on example 1.

rithm can solve 0-1 knapsack problems better than greedy algorithm, backtracking algorithm, dynamic programming algorithm and bound algorithm. These 0-1 knapsack problems of any two kinds of dispersion coefficient 


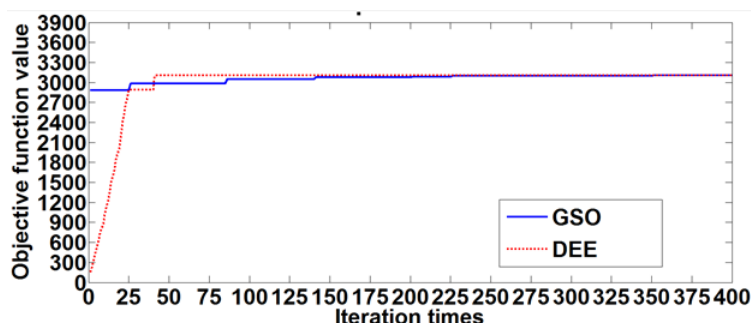

Figure 4. The convergence of GSO and DEE on example 3.

were in the same level, and all the three kinds of dispersion coefficient were less than 1 and any two kinds of dispersion coefficient were not in the same level. The research on discrete coefficient of data, which is a new idea this article explores, is the essence of this algorithm for verification.

\section{REFERENCES}

[1] A. A. Razborov, "On Provably Disjoint NP-Pairs. Electronic Colloquium on Computational Complexity,” Technical Report TR, 1994, pp. 94-006.

[2] E. Maslov, "Speeding up Branch and Bound Algorithms for Solving the Maximum Clique Problem," Journal of Global Optimization, 2013, pp. 1-12.

[3] H. Fouchal and Z. Habbas, "Distributed Backtracking Algorithm Based on Tree Decomposition over Wireless
Sensor Networks," Concurrency Computation Practice and Experience, Vol. 25, No. 5, 2013, pp. 728-742. http://dx.doi.org/10.1002/cpe.1804

[4] G. Lantoine, “A Hybrid Differential Dynamic Programming Algorithm for Constrained Optimal Control Problems," Journal of Optimization Theory and Applications, Vol. 154, No. 2, 2012, pp. 418-423. http://dx.doi.org/10.1007/s10957-012-0038-1

[5] F. Zhou, "A Particle Swarm Optimization Algorithm,” Applied Mechanics and Materials, 2013, pp. 1369-1372.

[6] K.-S. Yoo, “A Modified Ant Colony Optimization Algorithm for Dynamic Topology Optimization,” Computers and Structures, Vol. 123, 2013, pp. 68-78. http://dx.doi.org/10.1016/j.compstruc.2013.04.012

[7] Z. Y. Yan. "Exact Solutions of Nonlinear Dispersive $\mathrm{K}(\mathrm{m}$, n) Model with Variable Coefficients," Applied Mathematics and Computation, Vol. 217, No. 22, 2011, pp. 9474-9479. http://dx.doi.org/10.1016/j.amc.2011.04.047

[8] J. H. Lv, "The Experiment Data on 0-1 Knapsack Problem."

http://user.qzone.qq.com/1020052739/infocenter\#!app=2 \&via=QZ.HashRefresh\&pos=add

[9] K. Cheng and L. Ma, “Artificial Glowworm Swarm Optimization Algorithm for 0-1 Knapsack Problem,” Application Research of Computers, Vol. 30, No. 4, 2013, pp. 993-995. 